\title{
Case Series of Three Patients with Rifampicin-Induced Thrombocytopenia
}

\author{
Chandramouli M.T. ${ }^{1}$ \\ ${ }^{1}$ Department of Pulmonary Medicine, K.S. Hegde Medical Academy, \\ Mangalore, Karnataka, India
}

J Health Allied Sci Nu:2021;11:44-46

\begin{abstract}
Address for correspondence Chandramouli M.T., MD, Department of Pulmonary Medicine, K.S. Hegde Medical Academy, New Boys Hostel, KSHEMA Campus, Mangalore 575018, Karnataka, India (e-mail: mouli.aims@gmail.com).
\end{abstract}
Abstract
Keywords
- tuberculosis
- rifampicin
- thrombocytopenia
- purpura
- antiplatelet antibody

Life-threatening adverse reactions of antitubercular drugs are uncommon; however, thrombocytopenia is one such rare complication encountered with rifampicin, isoniazid, ethambutol, and pyrazinamide. Rifampicin is the most effective drug and its use in the tuberculosis treatment led to the emergence of modern and effective short-course regimens. I am reporting case series of three patients with pulmonary tuberculosis presented with rifampicin-induced thrombocytopenia.

\section{Introduction}

Antitubercular drugs are usually well tolerated. Prevalence of adverse reactions with first line antitubercular drugs varies from 8.0 to $85 \%$, most frequent adverse drug reactions are gastrointestinal upset, cutaneous reactions, and hepatotoxicity. ${ }^{1}$ Thrombocytopenia is uncommon but potentially life-threatening adverse effect seen with antitubercular drugs including rifampicin. ${ }^{2}$ Other rare but potentially serious adverse effects of rifampicin are hemolytic anemia, shock, and acute renal failure. ${ }^{3}$

The diagnosis of isolated thrombocytopenia in a patient taking several medications constitutes a challenging clinical problem. ${ }^{4}$ Laboratory confirmation of drug-induced thrombocytopenia at the time of initial presentation is not possible because tests for drug-dependent antiplatelet antibodies are not available in most clinical laboratories. ${ }^{5}$ Diagnosis of rifampicin-induced thrombocytopenia can be supported by resolution of thrombocytopenia after discontinuation of the suspected drug. Early detection with high index of suspicion and discontinuation of the offending drug is crucial in the management of drug-induced thrombocytopenia.

published online

October 16, 2020
DOI https://doi.org/

$10.1055 / \mathrm{s}-0040-1718608$

ISSN 2582-4287.

\section{Case Report}

\section{Case 1}

A 22-year-old male previously treated for tuberculosis diagnosed with sputum smear positive pulmonary tuberculosis was started on antitubercular therapy (ATT), fixed dose combination daily regimen of two tablets per day (isoniazid: $75 \mathrm{mg}$, rifampicin: $150 \mathrm{mg}$, ethambutol: $275 \mathrm{mg}$, pyrazinamide: $400 \mathrm{mg}$ ), 5 days later he presented with extensive purpura, gum bleeding, and hemoptysis.

On examination he had purpura all over the body and bleeding from gums, and had no lymphadenopathy or organomegaly. His platelet count was less than $10,000 / \mu \mathrm{L}$. ATT was stopped immediately, and platelets were transfused. One-week later platelet count returned to $166,000 / \mu \mathrm{L}$. After ruling out other causes of thrombocytopenia, ATT was reinitiated with one drug at a time, first isoniazid followed by ethambutol, and subsequently pyrazinamide was added. In the end rifampicin was added at dose of $450 \mathrm{mg} / \mathrm{day}$, but this again resulted in sudden drop in platelet count from $182,000 / \mu \mathrm{L}$ to $62,000 / \mu \mathrm{L}$. Rifampicin was stopped, and platelet count became normal after 6 days without any supportive treatment. Patient was discharged on isoniazid, ethambutol,

(C) 2020. Nitte (Deemed to be University)

This is an open access article published by Thieme under the terms of the Creative Commons Attribution-NonDerivative-NonCommercial-License, permitting copying and reproduction so long as the original work is given appropriate credit. Contents may not be used for commercial purposes, or adapted, remixed, transformed or built upon. (https://creativecommons.org/licenses/by-nc-nd/4.0/).

Thieme Medical and Scientific Publishers Pvt. Ltd. A-12, 2nd Floor, Sector 2, Noida-201301 UP, India 
pyrazinamide, streptomycin, and levofloxacin, there were no clinical and laboratory abnormalities noted during follow-up.

\section{Case 2}

A 47-year-old female presented with cough, loss of appetite, loss of weight for 1 month, hemoptysis, and purpura for 1 day. She was started on ATT category 2 (Cat-II) from primary health center for sputum smear positive pulmonary tuberculosis for 2 days. Sputum cartridge-based nucleic acid amplification test showed Mycobacterium tuberculosis which was detected to be rifampicin-sensitive.

On examination, she had purpura all over the body including the oral cavity (-Figs. 1 and 2 ), her platelet count was $7,000 / \mu \mathrm{L}$, hemoglobin ( $\mathrm{Hb}$ ) was $11.3 \mathrm{~g} / \mathrm{dL}$, renal and liver function test, bleeding time (BT), clotting time (CT), and prothrombin time-international normalized ratio (PT-INR) were within normal limits, dengue viral markers were negative, antinuclear antibody was negative, peripheral blood smear showed decreased platelet count, and normocytic normochromic red blood cells.

ATT was stopped immediately, and platelet transfusion was given, after 6 days platelet count became normal and stabilized. ATT with single-drug challenge was initiated. After isoniazid, ethambutol, and pyrazinamide challenge dose, rifampicin was added, this resulted in thrombocytopenia. Rifampicin was stopped, after which platelet count became normal after 5 days without any supportive treatment. She

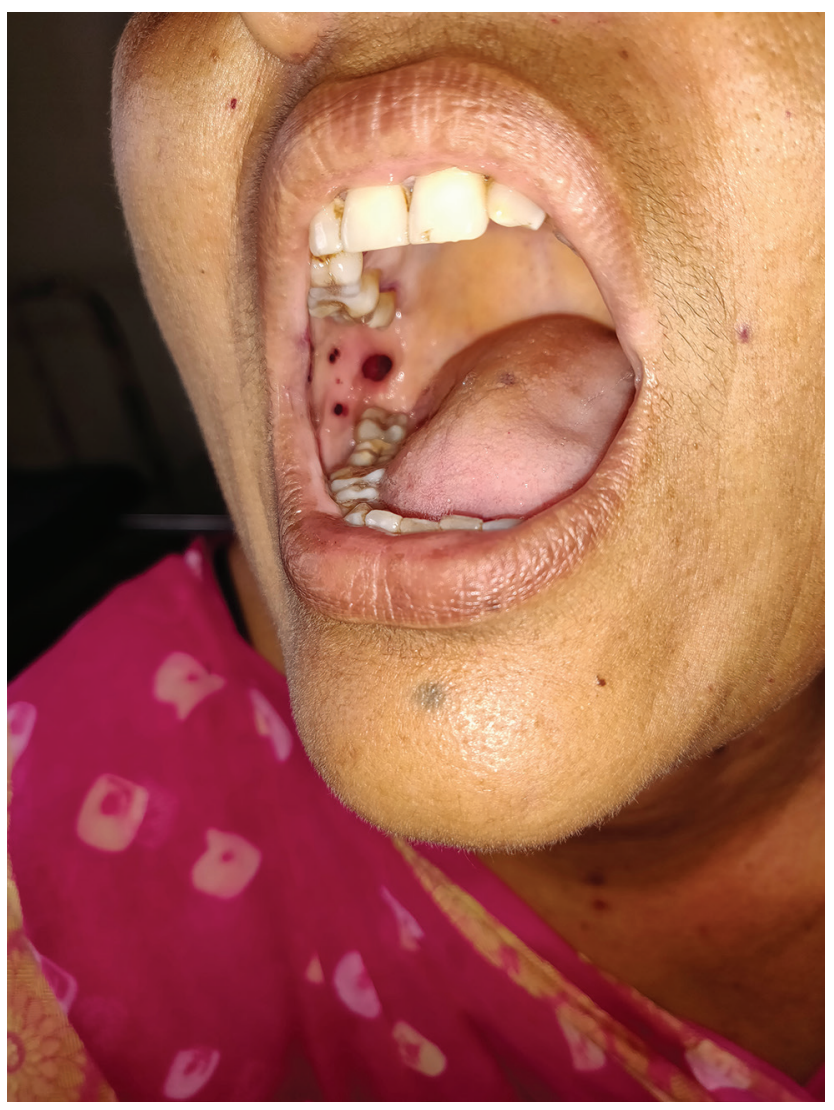

Fig. 1 Purpura on the right posterior buccal mucosa.

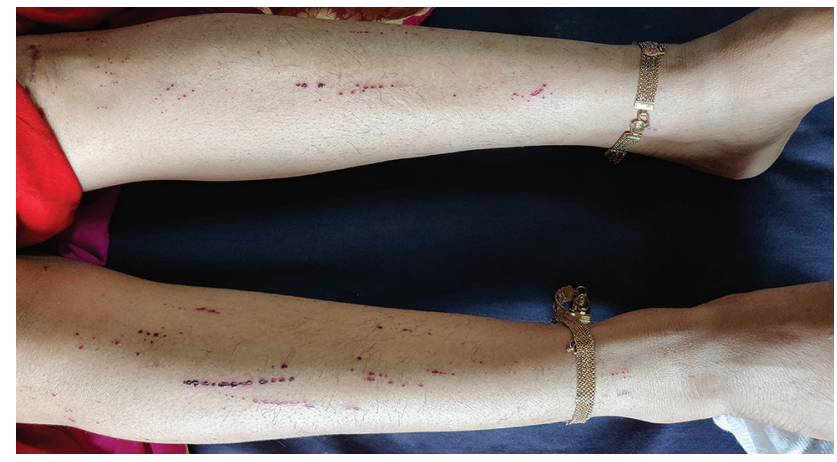

Fig. 2 Purpura on lower limbs.

has been counseled against taking rifampicin for the rest of her life.

\section{Case 3}

A 20-year-old male was admitted to the department of pulmonary medicine with complaints of cough, fever, loss of appetite for the last 2 months, and hemoptysis for 2 days. He was on ATT Cat-II for 20 days for sputum smear positive pulmonary tuberculosis from primary health center. On examination of the patient, revealed purpura all over the body, his

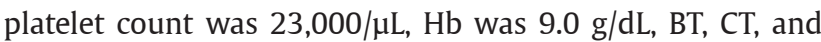
PT-INR were within normal limits, and dengue viral markers were negative.

ATT was stopped immediately, after 5 days his platelet count increased to $92,000 / \mu \mathrm{L}$ and purpura improved. After ruling out other causes of thrombocytopenia, ATT with single-drug challenge was initiated with isoniazid, ethambutol, and pyrazinamide with a gap of 5 days, lastly rifampicin was added, but this again resulted in low platelet count of $36,000 / \mu \mathrm{L}$. Rifampicin was stopped, after which platelet count became normal after 7 days. He was discharged on isoniazid, ethambutol, pyrazinamide, levofloxacin, and streptomycin and counseled against taking rifampicin for the rest of his life.

\section{Discussion}

Thrombocytopenia can occur with all first line antitubercular drugs (isoniazid, pyrazinamide, ethambutol, and rifampicin). In case of isoniazid, it occurs as hematological reaction. ${ }^{6}$ Thrombocytopenia due to ethambutol and pyrazinamide has also been reported, certainly by immunological mechanism. ${ }^{78}$ Other drugs associated with isolated thrombocytopenia are quinidine, quinine, heparin, sulfonamides, anticonvulsants (phenytoin, carbamazepine, ethosuximide, valproic acid), antibiotics (penicillins, cephalosporins, piperacillin, vancomycin, trimethoprim, levofloxacin, aztreonam, linezolid), nonsteroidal anti-inflammatory drugs (ibuprofen, aceclofenac, naproxen), abciximab, amiodarone, dexamethasone, furosemide, and gold compounds. ${ }^{9}$

Rifampicin is the most common antitubercular drug for the causation of thrombocytopenia. ${ }^{10}$ Rifampicin-induced thrombocytopenia involves antibody-mediated platelet destruction. ${ }^{11,12}$ Binding of drug to the platelet surface causes a conformational change in a surface protein that leads to 
exposure of a neo-epitope, which in turn stimulates the formation of antiplatelet antibodies. Antibodies bind noncovalently to specific platelet antigen via Fab regions.

In the present study, all three patients were previously treated for pulmonary tuberculosis with ATT intermittent regimen. Antitubercular drugs taken intermittently may cause immune-mediated thrombocytopenia even if it has been many years since the previous exposure.

Laboratory testing for drug-dependent antiplatelet antibodies can take several days and is not routinely available in most hospital laboratories. The antibody testing can be performed after the offending drug has been discontinued, because drug-dependent antiplatelet antibodies persist for many years. Positive test for drug-dependent antiplatelet antibodies confirms the diagnosis of drug-induced thrombocytopenia, while a negative test does not rule it out.

Rifampicin-induced thrombocytopenia should be managed with immediate drug discontinuation, platelet transfusion to maintain platelet count above $20,000 / \mu \mathrm{L}$, intravenous immunoglobulins, and corticosteroids may be administered initially when drug-induced thrombocytopenia is indistinguishable from primary idiopathic thrombocytopenic purpura.

Offending drug should not be reused as only a minute quantity of drug can lead to subsequent immune reaction.

\section{Conclusion}

In a tuberculosis endemic country like India, ATT is prescribed very frequently, sensitization of health care worker to rare yet lethal complications like thrombocytopenia helps in early detection and prompt intervention.

\section{Conflict of Interest}

None declared.

\section{References}

1 Shinde KM, Pore SM, Bapat TR. Adverse reactions to first-line anti-tuberculous agents in hospitalized patients: pattern, causality, severity and risk factors. Indian J Med Spec. 2013;4:1-4

2 George JN, Raskob GE, Shah SR, et al. Drug-induced thrombocytopenia: a systematic review of published case reports. Ann Intern Med 1998;129(11):886-890

3 Girling DJ, Hitze KL. Adverse reactions to rifampicin. Bull World Health Organ 1979;57(1):45-49

4 Wazny LD, Ariano RE. Evaluation and management of drug-induced thrombocytopenia in the acutely ill patient. Pharmacotherapy 2000;20(3):292-307

5 Di Berardino L, Perna G, Silvestri LG. Antibodies against rifampin in patients with tuberculosis after discontinuation of daily treatment. Am Rev Respir Dis 1976;114(6):1189-1190

6 Ross JD, Horne NW, Drugs used in chemotherapy. In: Horne NW, ed. Modern Drug Treatment of Tuberculosis. 1st Indian ed. New Delhi: Oxford University Press; 1992;1-17

7 Prasad R, Mukerji PK. Rifampicin induced thrombocytopenia. Indian J Tuberc 1989;36:44

8 Jain VK, Vardhan H, Prakash OM. Pyrazinamide induced thrombocytopenia. Tubercle 1988;69(3):217-218

9 Mitta A, Curtis BR, Reese JA, George JN. Drug-induced thrombocytopenia: 2019 update of clinical and laboratory data. Am J Hematol 2019;94(3):E76-E78

10 Garg R, Gupta V, Mehra S, Singh R, Prasad R. Rifampicin induced thrombocytopenia. Indian J Tuberc 2007;54(2):94-96

11 Blajchman MA, Lowry RC, Pettit JE, Stradling P. Rifampicininduced immune thrombocytopenia. BMJ 1970;3(5713): 24-26

12 Ackroyd JF. The pathogenesis of thrombocytopenic purpura due to hypersensitivity to sedormid. Clin Sci 1949;7(3-4):249-285 